\title{
NONASSOCIATIVE NORMED ALGEBRAS: GEOMETRIC ASPECTS
}

\author{
ANGEL RODRÍGUEZ PALACIOS
}

Departamento de Análisis Matemático, Facultad de Ciencias, Universidad de Granada

\author{
18071 Granada, Spain
}

Introduction. The aim of this paper is to review some relevant results concerning the geometry of nonassociative normed algebras, without assuming in the first instance that such algebras satisfy any familiar identity, like associativity, commutativity, or Jordan axiom. In the opinion of the author, the most impressive fact in this direction is that most of the celebrated natural geometric conditions that can be required for associative normed algebras, when imposed on a general nonassociative normed algebra, imply that the algebra is actually "nearly associative". We shall explain this idea by selecting four favourite topics, namely:

- Nonassociative Vidav-Palmer theorem,

- Nonassociative Gelfand-Naimark theorem,

- Nonassociative smooth normed algebras, and

- One-sided division absolute valued algebras.

Although there are classical nice forerunners in this circle of ideas, as for example the Albert-Urbanik-Wright determination of (nonassociative) absolute valued algebras with a unit ([2], [3], [42], and [41]), a systematic treatment of questions of this type has been made only recently, more precisely since 1980 [34].

1. Nonassociative Vidav-Palmer theorem. Recall that a normed algebra is called unital if it has a unit element $\mathbf{1}$ such that $\|\mathbf{1}\|=\mathbf{1}$, and that an element $h$ in a complex unital normed algebra $A$ is called hermitian if $\phi(h) \in \mathbb{R}$ for every continuous linear form $\phi$ on $A$ with $\|\phi\|=\phi(\mathbf{1})=\mathbf{1}$. For such an algebra $A$, we shall denote by $H(A)$ the closed real subspace of $A$ of all hermitian elements

1991 Mathematics Subject Classification: 46H70, 46K70.

The paper is in final form and no version of it will be published elsewhere. 
in $A$. The original associative Vidav-Palmer theorem (see [11; Theorem 6.9]) characterizes the unital associative $C^{*}$-algebras as those unital complete normed associative complex algebras $A$ satisfying $A=H(A)+i H(A)$ (associative Vidav algebras). Even in this case we have $H(A) \cap i H(A)=0$, and the $C^{*}$-algebra involution is nothing but the mapping $h+i k \rightarrow h-i k(h, k \in H(A))$. Now it is tempting to consider general nonassociative Vidav algebras as the nonassociative counterparts of unital associative $C^{*}$-algebras, expecting the appearance of many exotic $C^{*}$-models. Unexpectedly such nonassociative $C^{*}$-models are very close to their associative precursors. This will be explained in what follows, beginning with the so-called "nonassociative Vidav-Palmer theorem". This result attains its definitive form in [35] after a wide collection of papers, namely [10], [45], [46], [28], [34] and [26], and reads as follows.

THEOREM 1.1. Let $A$ be a unital complete normed nonassociative complex algebra satisfying $A=H(A)+i H(A)$. Then $A$ is a noncommutative Jordan algebra, $H(A) \cap i H(A)=0$, the mapping ${ }^{*}$ given by $(h+i k)^{*}:=h-i k(h, k \in H(A))$ is an algebra involution on $A$, and the equality $\left\|U_{a}\left(a^{*}\right)\right\|=\|a\|^{3}$ holds for every a in $A$ (where, for $a$ and $b$ in $\left.A, U_{a}(b):=(a b) a+(b a) a-b a^{2}\right)$.

Noncommutative Jordan algebras are defined as those nonassociative algebras satisfying the identities $(a b) a=a(b a)$ and $a^{2}(b a)=\left(a^{2} b\right) a$. Complete normed noncommutative Jordan complex algebras with an algebra involution * satisfying $\left\|U_{a}\left(a^{*}\right)\right\|=\|a\|^{3}$ for every $a$ in the algebra are called noncommutative $J B^{*}$ algebras. Since it is easy to show that unital noncommutative $J B^{*}$-algebras are Vidav algebras, the nonassociative Vidav-Palmer theorem asserts that general nonassociative Vidav algebras are nothing but noncommutative $J B^{*}$-algebras.

To understand in the first instance how close to associative $C^{*}$-algebras are noncommutative $J B^{*}$-algebras, take into account the associativity of powers of noncommutative Jordan algebras [38] to deduce easily that noncommutative $J B^{*}$ algebras are "locally" $C^{*}$-algebras, i.e. the closed subalgebra generated by any self-adjoint element is an associative and commutative $C^{*}$-algebra. A much better information can be derived from the structure theory developed in [32], [33], [5], and [13]. We shall summarize this theory in Theorems 1.2-1.4 below, following the lines of [32] and [33]. Given a family $\left\{A_{i}\right\}_{\in I}$ of noncommutative $J B^{*}$-algebras, any closed self-adjoint subalgebra $B$ of the noncommutative $J B^{*}$-algebra $\bigoplus_{i \in I}^{l_{\infty}} A_{i}$ with the property that $\pi_{i}(B)=A_{i}$ for all $i$ in $I$ (where $\pi_{i}$ denotes the natural projection onto the $i$ th coordinate) will be called a subdirect $l_{\infty}$-sum of the given family $\left\{A_{i}\right\}_{i \in I}$. Let us also say that a noncommutative $J B^{*}$-algebra $A$ is primitive if there exists an extreme point in the closed unit ball of its dual space whose kernel contains no nonzero ideals of $A$. An easy consequence of results by C. A. Akemann and B. Russo [1], explicitly stated in [32; Lemma 6.5], is that for associative $C^{*}$-algebras this concept of primitiveness agrees with the usual one. To be in agreement with the terminology in [33], note also that, thanks to [33; Corollary 1.13], primitive noncommutative $J B^{*}$-algebras as defined above are 
exactly the noncommutative $J B^{*}$-algebras having a faithful type I factor representation.

THEOREM 1.2. Every noncommutative $J B^{*}$-algebra is (totally isomorphic to) a subdirect $l_{\infty}$-sum of a suitable family of primitive noncommutative $J B^{*}$ algebras.

Because the noncommutative $J B^{*}$-algebras which are commutative were considered in the literature before the introduction of our general concept, they are simply called $J B^{*}$-algebras. Recall also that an algebra $A$ over a field $\mathbf{K}$ is called quadratic if it has a unit element $\mathbf{1}, A \neq \mathbf{K} \mathbf{1}$, and, for every $a$ in $A$, there are $\alpha, \beta$ in $\mathbf{K}$ such that $a^{2}+\alpha a+\beta \mathbf{1}=\mathbf{0}$.

THEOREM 1.3. The primitive noncommutative $J B^{*}$-algebras are the following:

(i) the primitive (commutative) $J B^{*}$-algebras,

(ii) the simple quadratic noncommutative $J B^{*}$-algebras, and

(iii) the noncommutative $J B^{*}$-algebras obtained from primitive associative $C^{*}$-algebras by changing the associative product " $a b$ " to $a \circ b:=\lambda a b+(1-\lambda) b a$, where $\lambda$ is a fixed real number with $0 \leq \lambda \leq 1$.

The simple quadratic noncommutative $J B^{*}$-algebras offer no problem because of the next result.

THEOREM 1.4. Given a real Hilbert space $E$ of dimension $>1$ with a bilinear anticommutative product $\wedge$ satisfying $(x \wedge y \mid z)=(x \mid y \wedge z)$ and $\|x \wedge y\| \leq\|x\|\|y\|$ for all $x, y, z$ in $E$, consider the real algebra $B$ whose vector space is $\mathbb{R} \mathbf{1} \oplus E$ and whose product is defined by

$$
(\alpha \mathbf{1}+x)(\beta \mathbf{1}+y):=[\alpha \beta-(x \mid y)] \mathbf{1}+\alpha y+\beta x+x \wedge y .
$$

Then the complexification of $B$, with the involution * defined by

$$
[\alpha \mathbf{1}+x+i(\beta \mathbf{1}+y)]^{*}:=\alpha \mathbf{1}-x-i(\beta \mathbf{1}-y),
$$

and norm given by

$$
\|b+i c\|^{2}:=\|b\|^{2}+\|c\|^{2}+2\left[\|b\|^{2}\|c\|^{2}-(b \mid c)^{2}\right]^{1 / 2}
$$

(where, in the right hand side of the equality, the inner product and norms refer to the natural hilbertian structure of $B$ as $l_{2}$-sum of $\mathbb{R} \mathbf{1}$ and $\left.E\right)$, is a simple quadratic noncommutative $J B^{*}$-algebra. Moreover, all simple quadratic noncommutative $J B^{*}$-algebras can be constructed in this way.

It follows from Theorems 1.2-1.4 that to have a complete structure theory for noncommutative $J B^{*}$-algebras, it only remains to describe the primitive $J B^{*}$ algebras. $J B^{*}$-algebras were introduced by J. D. M. Wright [43] who proved the existence of a natural one-to-one correspondence between the class of $J B^{*}$ algebras and the earlier studied class of $J B$-algebras (see [22] and references therein). In particular, he showed that the only finite-dimensional exceptional simple complex Jordan algebra $M_{3}^{8}(\mathbb{C})$ can be structured as a $J B^{*}$-algebra, and 
this in an essentially unique way. There is also a mutual dependence between $J B^{*}$-algebras and $J B^{*}$-triples (see $[40]$ and references therein), because $J B^{*}$ algebras can be naturally regarded as $J B^{*}$-triples and, conversely, $J B^{*}$-triples can be seen as $J B^{*}$-subtriples of $J B^{*}$-algebras [20; Corollary 2]. Concerning our interest in primitive $J B^{*}$-algebras, the appropriate description theorem has been obtained only very recently in [18] by mixing the classical $J B$ - and $J B^{*}$-theory with Zel'manov's techniques in the proof of his famous classification theorem for prime nondegenerate Jordan algebras (see [47] and [30]), and reads as follows.

THEOREM 1.5. The primitive $J B^{*}$-algebras are the following:

(i) $M_{3}^{8}(\mathbb{C})$,

(ii) the simple quadratic J $B^{*}$-algebras (given by Theorem 1.4 by taking $\wedge=0$ ),

(iii) the closed ${ }^{*}$-invariant Jordan subalgebras of $M(A)$ containing $A$, where $A$ is any primitive associative $C^{*}$-algebra, and $M(A)$ denotes the $C^{*}$-algebra of multipliers of $A$, and

(iv) the closed *-invariant Jordan subalgebras of $M(A)$ contained in $H(M(A), \tau)$ and containing $H(A, \tau)$, where $A$ is any primitive associative $C^{*}$ algebra, $\tau$ is $a^{*}$-involution on $A$ (linear algebra involution commuting with ${ }^{*}$ ), and $H(\cdot, \tau)$ denotes the set of all $\tau$-invariant elements.

Remark 1.1. Following an earlier idea by E. Zel'manov in the case of the existence of a unit, a purely algebraic concept of primitiveness for Jordan algebras was introduced and studied by L. Hogben and K. McCrimmon [23], and later this concept was extended to the setting of noncommutative Jordan algebras [19]. In the original commutative context this notion has been successfully revisited in two recent papers by J. A. Anquela, F. Montaner, and T. Cortés ([6] and [7]) from the point of view of Zel'manov's methods. It follows from Theorem 1.5 and results in [6] that, for noncommutative $J B^{*}$-algebras, the algebraic concept of primitiveness agrees with that we have taken in this survey.

2. Nonassociative Gelfand-Naimark theorem. Another reasonable approach to nonassociative counterparts of associative $C^{*}$-algebras may arise by the consideration of complete normed nonassociative complex algebras with a conjugate-linear algebra involution * satisfying the Gelfand-Naimark axiom $\left\|a^{*} a\right\|$ $=\|a\|^{2}$. It is the aim of this section to review the results that are known in this direction, showing that, in the case of the existence of a unit, these Gelfand-Naimark nonassociative algebras are very particular cases of the above considered Vidav nonassociative algebras. The fundamental result in this direction was obtained in [34] using a "light" version of Theorem 1.1, earlier proved in [26], together with a result in [44] on isometries of $J B$-algebras.

TheOREM 2.1. Let $A$ be a complete normed nonassociative complex algebra with a unit $\mathbf{1}$ and a conjugate-linear vector space involution ${ }^{*}$ satisfying $\mathbf{1}^{*}=\mathbf{1}$ 
and $\left\|a^{*} a\right\|=\|a\|^{2}$ for every $a$ in $A$. Then $A$ is an alternative algebra, and ${ }^{*}$ is an algebra involution on $A$.

We recall that an algebra $A$ is said to be alternative if the equalities $a^{2} b=$ $a(a b)$ and $b a^{2}=(b a) a$ hold for all $a, b$ in $A$, and that this requirement is equivalent (via Artin's theorem [38]) to the fact that the subalgebra of $A$ generated by two arbitrary elements in $A$ is associative. Complete normed alternative complex algebras with an algebra involution * satisfying $\left\|a^{*} a\right\|=\|a\|^{2}$ for every $a$ in the algebra are called alternative $C^{*}$-algebras. Alternative algebras are noncommutative Jordan algebras (use Artin's theorem), and it is easy to see that alternative $C^{*}$-algebras are in fact noncommutative $J B^{*}$-algebras. More precisely, alternative $C^{*}$-algebras are exactly those noncommutative $J B^{*}$-algebras which are alternative. Thus we may apply Theorem 1.2 to obtain the following corollary.

Corollary 2.1. Every alternative $C^{*}$-algebra is the subdirect $l_{\infty}$-sum of a suitable family of primitive alternative $C^{*}$-algebras.

The algebra of complex octonions can be structured in an essentially unique way as an alternative $C^{*}$-algebra $([26]$ and $[14])$, and this alternative $C^{*}$-algebra is primitive because it is actually simple. In fact we have the following theorem (see [32] and [14]), which today can be easily derived from Theorem 1.3.

THEOREM 2.2. Every primitive alternative $C^{*}$-algebra is either associative or the $C^{*}$-algebra of complex octonions.

Complete normed nonassociative complex algebras with a unit $\mathbf{1}$ and a conjugate-linear algebra involution * satisfying $\left\|a^{*} a\right\|=\left\|a^{*}\right\|\|a\|$ are nothing but unital alternative $C^{*}$-algebras [34]. In this "weak" Gelfand-Naimark situation it is tempting to consider the question of the possibility of relaxing the requirement $(a b)^{*}=b^{*} a^{*}$ to the weak one $\mathbf{1}^{*}=\mathbf{1}$, without perturbing the characterization of alternative $C^{*}$-algebras (in the same way as this has been possible in the "strong" Gelfand-Naimark situation studied in Theorem 2.1). Actually, the answer to this question is "almost" affirmative, as follows from the next theorem, that has been proved very recently in [16] using as main tools Theorems 1.1, 1.4, and 2.1.

THEOREM 2.3. Let $A$ be a complete normed complex nonassociative algebra with a unit $\mathbf{1}$ and a conjugate-linear vector space involution $\square$ satisfying $\mathbf{1}^{\square}=\mathbf{1}$ and $\left\|a^{\square} a\right\|=\left\|a^{\square}\right\|\|a\|$ for every $a$ in $A$. Then $A$ is an alternative algebra, and (except possibly in the case when $A$ is isometrically isomorphic to the $C^{*}$-algebra $\left.\mathbb{C}^{2}\right) \square$ is an algebra involution on $A$ and the equality $\left\|a^{\square} a\right\|=\|a\|^{2}$ holds for every a in $A$. For the exceptional case of the $C^{*}$-algebra $\mathbb{C}^{2}$, the involutions $\square$ satisfying the above requirements are exactly the mappings of the form $a \rightarrow a^{*}+\bar{f}(a) \mathbf{1}$, where * denotes the $C^{*}$-algebra involution and $f$ is any fixed linear form on $\mathbb{C}^{2}$ such that $f(\mathbf{1})=\mathbf{0}$ and $f\left(a^{*}\right)=-\bar{f}(a)$ for all $a$ in $\mathbb{C}^{2}$.

Remark 2.1. In the case of absence of a unit element, complete normed nonassociative complex algebras with a conjugate-linear algebra involution * sat- 
isfying $\left\|a^{*} a\right\|=\|a\|^{2}$ need not satisfy identities. To see this consider a nonempty set $V$, let $B$ denote the free nonassociative complex algebra on $V[24 ;$ p. 23], and let ${ }^{*}$ be the unique conjugate-linear algebra involution on $B$ fixing the elements of $V$. Since the vector space of $B$ is the free complex space on the set $U$ of all "nonassociative words" with entries in $V$, we may endow $B$ with the norm $\|\cdot\|$ given by either

$$
\left\|\sum_{u \in U} \lambda_{u} u\right\|:=\left(\sum_{u \in U}\left|\lambda_{u}\right|^{p}\right)^{1 / p}
$$

( $p$ any real number with $p \geq 1$ ), or

$$
\left\|\sum_{u \in U} \lambda_{u} u\right\|:=\sup \left\{\left|\lambda_{u}\right|: u \in U\right\} .
$$

Then, for $b$ and $c$ in $B$, we have $\|b c\|=\|b\|\|c\|$ (see [15; Introduction] and [37; Remarks 3(i) and 3(ii)]) and $\left\|b^{*}\right\|=\|b\|$. Now, denoting by $A$ the completion of $(B,\|\cdot\|)$, and extending * to $A$ by continuity, $A$ becomes a complete normed nonassociative complex algebra with a conjugate-linear algebra involution ${ }^{*}$ satisfying $\left\|a^{*} a\right\|=\|a\|^{2}$ for all $a$ in $A$. Moreover, if the set $V$ is infinite, clearly $A$ cannot satisfy any identity.

3. Nonassociative smooth normed algebras. Recall that a smooth normed algebra is a unital normed algebra whose unit $\mathbf{1}$ is a smooth point of its closed unit ball, i.e. there is a unique element $\phi$ in the dual space of the given normed algebra with $\|\phi\|=\phi(\mathbf{1})=1$. It is well known that $\mathbb{C}$ is the only smooth normed nonassociative complex algebra, as well as that $\mathbb{R}, \mathbb{C}$, and $\mathbb{H}$ (the division algebra of real quaternions), with their usual absolute values as norms, are the only smooth normed associative real algebras. J. I. Nieto [31] determined the smooth normed alternative real algebras, showing that they are exactly $\mathbb{R}, \mathbb{C}, \mathbb{H}$, and $\mathbb{O}$ (the division algebra of real octonions). Nieto's proof involves earlier results by E. Strzelecki [39] on smooth normed power-associative real algebras. But actually general nonassociative smooth normed real algebras are noncommutative Jordan algebras (hence power-associative), as shown by the following theorem that was proved in [35] as a relatively easy consequence of Theorem 1.1 (see also [36] for a more direct proof not involving Theorem 1.1).

THEOREM 3.1. Given a real pre-Hilbert space $E$ with a bilinear anticommutative product $\wedge$ satisfying $(x \wedge y \mid z)=(x \mid y \wedge z)$ and $\|x \wedge y\| \leq\|x\|\|y\|$ for all $x, y, z$ in $E$, consider the real normed space $B=\mathbb{R} \mathbf{1} \oplus^{l_{2}} E$ with product defined by

$$
(\alpha \mathbf{1}+x)(\beta \mathbf{1}+y):=[\alpha \beta-(x \mid y)] \mathbf{1}+\alpha y+\beta x+x \wedge y .
$$

Then $B$ is a smooth normed algebra. Moreover, all smooth normed real algebras are of this type.

An almost direct consequence of this theorem is the following characterization of real pre-Hilbert spaces. 
Corollary 3.1. Let $X$ be a nonzero real normed space. Then the following assertions are equivalent:

(i) $X$ is a pre-Hilbert space.

(ii) If $u$ is any norm-one element in $X$, then $X$ is smooth at $u$ and the set of continuous bilinear mappings $f: X \times X \rightarrow X$, satisfying $\|f\|=1$ and $f(x, u)=$ $f(u, x)=x$ for all $x$ in $X$, is nonempty.

(iii) There is a norm-one element $u$ in $X$ such that $X$ is smooth at $u$ and the set of continuous bilinear mappings $f: X \times X \rightarrow X$, satisfying $\|f\|=1$ and $f(x, u)=f(u, x)=x$ for all $x$ in $X$, is nonempty.

In fact, the above characterization of real pre-Hilbert spaces can be improved in such a way that it only involves "numerical" conditions. This is a previously unpublished result of the author, whose statement and proof are included in what follows.

Given a normed space $X$ and a norm-one element $u$ in $X$, let us define the multiplicative index $m(X, u)$ of $X$ at $u$ as the infimum of the norms of the continuous bilinear mappings $f: X \times X \rightarrow X$ satisfying $f(x, u)=f(u, x)=x$ for all $x$ in $X$.

THEOREM 3.2. Let $X$ be a nonzero real normed space. Then the following assertions are equivalent:

(i) $X$ is a pre-Hilbert space.

(ii) If $u$ is any norm-one element in $X$, then $X$ is smooth at $u$ and $m(X, u)=1$.

(iii) There is a norm-one element $u$ in $X$ such that $X$ is smooth at $u$ and $m(X, u)=1$.

Proof. In view of Corollary 3.1, it is enough to show that (iii) implies (i). Therefore let us assume that (iii) holds, so that there is a sequence $\left\{f_{n}\right\}$ of continuous bilinear mappings from $X \times X$ into $X$ satisfying $\left\{\left\|f_{n}\right\|\right\} \rightarrow 1$ and $f_{n}(x, u)=f_{n}(u, x)=x$ for all $x$ in $X$ and $n$ in $\mathbb{N}$. By passing to third Arens adjoints, we obtain a sequence $\left\{g_{n}\right\}$ of continuous bilinear mappings from $X^{* *} \times X^{* *}$ into $X^{* *}$ satisfying $\left\{\left\|g_{n}\right\|\right\} \rightarrow 1$ and $g_{n}(z, u)=g_{n}(u, z)=z$ for all $z$ in $X^{* *}$ and $n$ in $\mathbb{N}$.

Now denote by $Y$ the Banach space of all continuous bilinear mappings from $X^{* *} \times X^{* *}$ into $X^{* *}$, and endow $Y$ with the topology $\tau$ of pointwise convergence in the $w^{*}$-topology of $X^{* *}$. It is well known and easy to see that closed balls in $Y$ are $\tau$-compact, hence there exists a $\tau$-point-limit $g$ for the sequence $\left\{g_{n}\right\}$, and clearly $\|g\| \leq 1$ and $g(z, u)=g(u, z)=z$ for all $z$ in $X^{* *}$. In this way $X^{* *}$, with the product $g$, becomes a unital (complete) normed algebra with unit $u$, so, by [29; Proposition 4.5], the duality mapping of $X^{* *}$ is (norm) $\times($ norm) upper semicontinuous at $u$ in the sense of [21]. Now, since $X$ is smooth at $u$, it is enough to apply [8; Theorems 5.1 and 3.4] to conclude that $X^{* *}$ is smooth at $u$. (Alternatively, we may use the fact that (norm) $\times$ (norm) upper semicontinuity of the duality mapping is inherited by subspaces, and implies $($ norm $) \times($ weak $)$ 
upper semicontinuity, to derive that the duality mapping of the completion of $X$ is $($ norm $) \times($ weak $)$ upper semicontinuous at $u$, and then we may apply [21; Theorem 3.1] to deduce as well that $X^{* *}$ is smooth at $u$ ). By the implication (iii) $\Rightarrow$ (i) in Corollary 3.1, $X^{* *}$ is a Hilbert space, hence $X$ is a pre-Hilbert space.

Recall that an absolute valued algebra is a nonzero algebra $A$ with a norm $\|\cdot\|$ satisfying $\|a b\|=\|a\|\|b\|$ for all $a, b$ in $A$. In Remark 2.1 we have seen examples of infinite-dimensional absolute valued complex algebras. It is easy to show that absolute valued algebras with a unit are smooth normed algebras. Then we may use Theorem 3.1 to derive that absolute valued real algebras with a unit are precisely those smooth normed real algebras that are alternative. Since also from Theorem 3.1 it follows that these alternative algebras are quadratic division algebras, it is enough to apply the extended Frobenius theorem (see for example [22; Theorem 2.26]), to obtain the following corollary, in which we collect the Albert-Urbanik-Wright theorem, already quoted in the introduction, as well as the above mentioned result by J. I. Nieto.

COROLlary 3.2. For a real algebra A, the following assertions are equivalent:

(i) $A$ is an absolute valued algebra with a unit.

(ii) $A$ is a smooth normed alternative algebra.

(iii) A equals $\mathbb{R}, \mathbb{C}, \mathbb{H}$, or $\mathbb{O}$, with its usual absolute value as norm.

4. One-sided division absolute valued algebras. By a left division algebra we mean a nonzero algebra (say $A$ ) with the property that, whenever $a$ and $b$ are in $A$ with $a \neq 0$, there exists a unique $x$ in $A$ satisfying $a x=b$. Of course we can consider the analogous concept of a right division algebra, and we say that $A$ is a division algebra if it is at the same time a left division and a right division algebra. A still unsolved old question is that of the nonassociative extension of the Gelfand-Mazur theorem, namely if any division normed (nonassociative) algebra must be finite-dimensional (which would imply dimension 1 in the complex case, and 1, 2, 4, 8 in the real one, by a theorem of R. Bott and J. Milnor [12]). This problem was explicitly posed by F. B. Wright [42] in 1953, who in the same paper gave a partial affirmative answer proving that division absolute valued algebras are finite-dimensional. Another folklore partial positive result about this question is that one-sided division complete normed complex algebras are isomorphic to the complex field, the case of (two-sided) division noncomplete normed complex algebras as well as that of division (even complete) normed real algebras remaining open.

In the converse direction, J. A. Cuenca [17] and the author [37] have proved very recently and almost at the same time the existence of one-sided division infinite-dimensional complete absolute valued real algebras. The Banach spaces of the algebras in these examples are in fact Hilbert spaces, of hilbertian dimension $\aleph_{0}$ in Cuenca's nice construction, and of arbitrary infinite hilbertian dimension in the more involved construction by the author. Since the fact that one-sided 
division absolute valued complex algebras are isomorphic to the complex field can be considered as folklore, it seems to be reasonable to look for a structure theory of arbitrary one-sided division absolute valued real algebras. Such a structure theory is provided in [37], and will be reviewed in what follows.

A first easy result in this direction is given by the next proposition involving a peculiar concept of "isotopy" between absolute valued algebras. Two absolute valued algebras $A$ and $B$ are said to be isotopic if there exist linear isometries $\varphi_{1}, \varphi_{2}, \varphi_{3}$ from $A$ onto $B$ satisfying

$$
\varphi_{1}(x y)=\varphi_{2}(x) \varphi_{3}(y)
$$

for all $x, y$ in $A$. This notion is motivated by the obvious fact that, given an absolute valued algebra $A$ and arbitrary linear isometries $\varphi_{1}, \varphi_{2}, \varphi_{3}$ from $A$ onto $A$, the normed space of $A$ with the new product $\odot$ given by

$$
x \odot y:=\varphi_{1}^{-1}\left(\varphi_{2}(x) \varphi_{3}(y)\right)
$$

becomes an absolute valued algebra.

Proposition 4.1. An absolute valued algebra is a left division algebra if and only if it is isotopic to a left division absolute valued algebra with a left unit.

Now the attention must be centered on absolute valued real algebras with a left unit, and then the set (say $P$ ) of all left multiplication operators on such an algebra is a space of bounded linear operators on the normed space ( say $X$ ) of the algebra containing the identity operator and satisfying

$$
\|T(x)\|=\|T\|\|x\|
$$

for all $T$ in $P$ and $x$ in $X$. We isolate this information, that has its own interest, and we obtain the following theorem.

THEOREM 4.1. Let $X$ be a nonzero real normed space, and $P$ be a space of bounded linear operators on $X$ containing the identity operator (say I) and satisfying

$$
\|T(x)\|=\|T\|\|x\|
$$

for all $T$ in $P$ and all $x$ in $X$. Then the operator norm on $P$ derives from an inner product and, for $T$ in $P$ orthogonal to $I$, the equality

$$
T^{2}=-\|T\|^{2} I
$$

holds.

As a first consequence, in the setting of the theorem every nonzero element in $P$ is an invertible operator on $X$. This fact reflects for absolute valued algebras in the following corollary.

COROLLARY 4.1. Every absolute valued algebra with a left unit is a left division algebra.

It follows also from the theorem that the space $P$ of operators considered above is actually a Jordan algebra of bounded linear operators on $X$, and that 
more precisely, if we denote by $(\cdot \mid \cdot)$ the inner product from which the operator norm on $P$ derives, then we have

$$
(\lambda I+S) \cdot(\mu I+T)=(\lambda \mu-(S \mid T)) I+\lambda T+\mu S,
$$

for all $\lambda, \mu$ in $\mathbb{R}$ and $S, T$ in $P$ orthogonal to $I$ (where "." denotes Jordan product, namely $\left.F . G:=\frac{1}{2}(F G+G F)\right)$. Considering the description of smooth normed real algebras given by Theorem 3.1 , particularizing to the commutative case $(\wedge=0)$, and calling these algebras "smooth normed Jordan algebras" (they are actually Jordan algebras), Theorem 4.1 can be reformulated as follows.

THEOREM 4.1 (bis). Let $X$ be a nonzero real normed space, and $P$ be a space of bounded linear operators on $X$ containing the identity operator and satisfying

$$
\|T(x)\|=\|T\|\|x\|
$$

for all $T$ in $P$ and all $x$ in $X$. Then $P$ is a Jordan algebra of operators on $X$ which, endowed with the operator norm, is isometrically isomorphic to a smooth normed Jordan algebra.

From this version of Theorem 4.1 the structure of (automatically left division) absolute valued algebras with a left unit follows easily by introducing some natural terminology. Given a Jordan algebra $J$ and a vector space $X$, a representation of $J$ on $X$ will mean a homomorphism (say $\psi$ ) from $J$ onto a Jordan algebra of operators on $X$. If $J$ has a unit $\mathbf{1}$ and $\psi(\mathbf{1})$ equals the identity operator on $X$, the representation $\psi$ will be called unital. If $X$ is a pre-Hilbert space, ${ }^{*}$ is an algebra involution on $J$, and the representation $\psi$ satisfies

$$
(\psi(x)(\eta) \mid \xi)=\left(\eta \mid \psi\left(x^{*}\right)(\xi)\right)
$$

for all $x$ in $J$ and all $\eta, \xi$ in $X$, then we will say that $\psi$ is a ${ }^{*}$-representation. Every smooth normed Jordan algebra $J=\mathbb{R} 1 \oplus E$ will be considered as an algebra with involution * defined by

$$
(\lambda \mathbf{1}+\eta)^{*}:=\lambda \mathbf{1}-\eta
$$

This involution can be intrinsically characterized as the only algebra involution * in $J$ such that, for every $x$ in $J, x+x^{*}$ and $x . x^{*}$ lie in $\mathbb{R} \mathbf{1}$.

THEOREM 4.2. If $J$ is a smooth normed Jordan algebra and $\psi$ is a unital *-representation of $J$ on the pre-Hilbert space of $J$, then the normed space of $J$ with product $\odot$ defined by

$$
x \odot y:=\psi(x)(y)
$$

is an (automatically left division) absolute valued algebra with left unit. Moreover, up to isometric isomorphism, all absolute valued algebras with left unit can be constructed in this way.

Remarks 4.1. (i) The first assertion in Theorem 4.2 is easy to prove, and has become the common starting idea in [17] and [37] for the construction of the infinite-dimensional examples of left-division absolute valued algebras commented on at the beginning of this section. Concerning the last assertion in Theorem 4.2 
(the most relevant), it can be stated without involving Jordan algebras and their representations on vector spaces, as follows. The norm of any absolute valued algebra $A$ with a left unit $e$ derives from an inner product $(\cdot \mid \cdot)$, and, for $a, b, c$ in $A$ with a orthogonal to e, we have

$$
(a b \mid c)=-(b \mid a c) \quad \text { and } \quad a(a b)=-\|a\|^{2} b .
$$

(ii) The Albert-Urbanik-Wright theorem on absolute valued algebras with unit, already quoted in Corollary 3.2, can also be easily derived from the above remark. For, if $A$ is such an algebra and $\mathbf{1}$ denotes its unit element, taking $b=\mathbf{1}$ in the last equality we obtain $a^{2}=-\|a\|^{2} \mathbf{1}$ for all $a$ in $A$ orthogonal to $\mathbf{1}$, hence $A$ is a quadratic algebra. Moreover, the same equality now yields $a(a b)=a^{2} b$, for $a$ and $b$ in $A$ with $a$ orthogonal to $\mathbf{1}$, and by symmetry we also have ( $b a) a=b a^{2}$, hence $A$ is alternative. Now $A$ is a division quadratic alternative algebra, so it is isomorphic to $\mathbb{R}, \mathbb{C}, \mathbb{H}$, or $\mathbb{O}$ by the extended Frobenius theorem.

(iii) Contrarily to what has happened for the remaining geometric requirements we have considered for nonassociative normed algebras in this paper, infinite-dimensional absolute valued algebras cannot satisfy any familiar identity like associativity or commutativity. While the result concerning associativity is a consequence of the Gelfand-Mazur-Kaplansky theorem on associative normed algebras with no nonzero zero-topological-divisors, the one concerning commutativity is due to K. Urbanik and F. B. Wright [41]. Both results are contained in the one by M. L. El-Mallah and A. Micali [27] asserting that an absolute valued algebra satisfying the identity $a(b a)=(a b) a$ is finite-dimensional. However, although infinite-dimensional left-division absolute valued algebras cannot be noncommutative Jordan algebras, by means of Proposition 4.1 and Theorem 4.2 their structure is reduced to that of some very particular normed Jordan algebras.

5. Concluding remarks. There are other relevant results of the kind considered in this talk, but that we have not mentioned explicitly. We shall conclude with a list of such results, all concerning nonassociative characterizations of some associative normed algebras. Thus we know:

- The nonassociative characterization of the real Banach algebras $\mathcal{C}_{\mathbb{R}}(K)$ ( $K$ a compact Hausdorff topological space) by R. V. Kadison [25].

- The nonassociative characterization of the complex Banach algebras $\mathcal{C}_{\mathbb{C}}(K)$ [34; Corollary 32].

- The nonassociative characterization of unital associative $C^{*}$-algebras. From the results in [32; Section 6] this characterization can be provided by assuming additionally in our Theorem 2.1 that the Banach space of the nonassociative Gelfand-Naimark algebra $A$ has no "primitive $M$-ideals" (in the sense of [4]) of codimension 8 .

- The nonassociative characterization of unital nonself-adjoint algebras of bounded linear operators on a complex Hilbert space, by D. P. Blecher, Z. Ruan, and A. M. Sinclair [9]. With the associative Vidav-Palmer theorem, this result 
contains a new nonassociative characterization of unital associative $C^{*}$-algebras [9; Corollary 3.3].

\section{References}

[1] C. A. Akemann and B. Russo, Geometry of the unit sphere of a $C^{*}$-algebra and its dual, Pacific J. Math. 32 (1970), 575-585.

[2] A. A. Albert, Absolute valued algebras, Ann. of Math. 48 (1947), 495-501.

[3] —, Absolute valued algebraic algebras, Bull. Amer. Math. Soc. 55 (1949), 763-768; A note of correction, ibid. 55 (1949), 1191

[4] E. M. Alfsen and E. G. Effros, Structure in real Banach spaces II, Ann. of Math. 96 (1972), 129-173.

[5] K. Alvermann and G. Janssen, Real and complex non-commutative Jordan Banach algebras, Math. Z. 185 (1984), 105-113.

[6] J. A. Anquela, F. Montaner, and T. Cortés, On primitive Jordan algebras, J. Algebra, to appear.

[7] - - - - On maximal modular inner ideals in Jordan algebras, Comm. Algebra 21 (1993), $2537-2554$.

[8] C. Aparicio, F. Ocaña, R. Payá, and A. Rodríguez, A non-smooth extension of Fréchet differentiability of the norm with applications to numerical ranges, Glasgow Math. J. 28 (1986), 121-137.

[9] D. B. Blecher, Z. Ruan, and A. M. Sinclair, A characterization of operator algebras, J. Funct. Anal. 89 (1990), 188-201.

[10] F. F. Bonsall, Jordan algebras spanned by hermitian elements of a Banach algebra, Math. Proc. Cambridge Philos. Soc. 81 (1977), 3-13.

[11] F. F. Bonsall and J. Duncan, Numerical Ranges of Operators on Normed Spaces and of Elements of Normed Algebras, London Math. Soc. Lecture Note Ser. 2, Cambridge University Press, 1971.

[12] R. Bott and J. Milnor, On the parallelizability of the spheres, Bull. Amer. Math. Soc. 64 (1958), 87-89.

[13] R. B. Braun, Structure and representations of non-commutative $C^{*}$-Jordan algebras, Manuscripta Math. 41 (1983), 139-171.

[14] _, A Gelfand-Neumark theorem for $C^{*}$-alternative algebras, Math. Z. 185 (1984), 225242.

[15] M. Cabrera and A. Rodríguez, Nonassociative ultraprime normed algebras, Quart. J. Math. Oxford 43 (1992), 1-7.

[16] _, - - New associative and nonassociative Gelfand-Naimark theorems, Manuscripta Math. 79 (1993), 197-208

[17] J. A. Cuenca, On one-sided division infinite-dimensional normed real algebras, Publ. Mat. 36 (1992), 485-488.

[18] A. Fernández, E. Garcia, and A. Rodríguez, A Zel'manov prime theorem for JB*algebras, J. London Math. Soc. 46 (1992), 319-335.

[19] A. Fernández and A. Rodríguez, Primitive noncommutative Jordan algebras with nonzero socle, Proc. Amer. Math. Soc. 96 (1986), 199-206.

[20] Y. Friedman and B. Russo, The Gelfand-Naimark theorem for JB*-triples, Duke Math. J. 53 (1986), 139-148.

[21] J. R. Giles, D. A. Gregory, and B. Sims, Geometrical implications of upper semicontinuity of the duality mapping on a Banach space, Pacific J. Math. 79 (1978), 99-108. 
[22] H. Hanche-Olsen and E. Stormer, Jordan Operator Algebras, Monograph Stud. Math. 21, Pitman, 1984.

[23] L. Hogben and K. McCrimmon, Maximal modular inner ideals and the Jacobson radical of a Jordan algebra, J. Algebra 68 (1981), 155-169.

[24] N. Jacobson, Structure and Representations of Jordan Algebras, Amer. Math. Soc. Colloq. Publ. 39, Providence, R.I., 1968.

[25] R. V. Kadison, A representation theory for commutative topological algebras, Mem. Amer. Math. Soc. 7 (1951).

[26] A. M. Kaidi, J. Martínez, and A. Rodríguez, On a nonassociative Vidav-Palmer theorem, Quart. J. Math. Oxford 32 (1981), 435-442.

[27] M. L. El-Mallah et A. Micali, Sur les dimensions des algèbres absolument valuées, J. Algebra 68 (1981), 237-246.

[28] J. Martínez, JV-algebras, Math. Proc. Cambridge Philos. Soc. 87 (1980), 47-50.

[29] J. Martínez, J. F. Mena, R. Payá, and A. Rodríguez, An approach to numerical ranges without Banach algebra theory, Illinois J. Math. 29 (1985), 609-626.

[30] K. McCrimmon and E. Zel'manov, The structure of strongly prime quadratic Jordan algebras, Adv. in Math. 69 (1988), 133-222.

[31] J. I. Nieto, Gateaux differentials in Banach algebras, Math. Z. 139 (1974), 23-34.

[32] R. Payá, J. Pérez, and A. Rodríguez, Non-commutative Jordan $C^{*}$-algebras, Manuscripta Math. 37 (1982), 87-120.

[33] - , - - , Type I factor representations of non-commutative $J B^{*}$-algebras, Proc. London Math. Soc. 48 (1984), 428-444.

[34] A. Rodríguez, A Vidav-Palmer theorem for Jordan $C^{*}$-algebras and related topics, J. London Math. Soc. 22 (1980), 318-332.

[35] —, Nonassociative normed algebras spanned by hermitian elements, Proc. London Math. Soc. 47 (1983), 258-274.

[36] - An approach to Jordan-Banach algebras from the theory of nonassociative complete normed algebras, Ann. Sci. Univ. Clermont-Ferrand II Math. 27 (1991), 1-57.

[37] —, One-sided division absolute valued algebras, Publ. Mat. 36 (1992), 925-954.

[38] R. D. Schafer, An Introduction to Nonassociative Algebras, Academic Press, New York, 1966.

[39] E. Strzelecki, Power-associative regular real normed algebras, J. Austral. Math. Soc. 6 (1966), 193-209.

[40] H. Upmeier, Symmetric Banach Manifolds and Jordan C $^{*}$-algebras, North-Holland, Amsterdam, 1985.

[41] K. Urbanik and F. B. Wright, Absolute valued algebras, Proc. Amer. Math. Soc. 11 (1960), 861-866.

[42] F. B. Wright, Absolute valued algebras, Proc. Nat. Acad. Sci. U.S.A. 39 (1953), 330-332.

[43] J. D. M. Wright, Jordan $C^{*}$-algebras, Michigan Math. J. 24 (1977), 291-302.

[44] J. D. M. Wright and M. A. Youngson, On isometries of Jordan algebras, J. London Math. Soc. 17 (1978), 339-344.

[45] M. A. Youngson, A Vidav theorem for Banach Jordan algebras, Math. Proc. Cambridge Philos. Soc. 84 (1978), 263-272.

[46] - Hermitian operators on Banach Jordan algebras, Proc. Edinburgh Math. Soc. 22 (1979), 93-104.

[47] E. Zel'manov, On prime Jordan algebras II, Siberian Math. J. 24 (1983), 89-104. 